\title{
PROYECTO DE INTERVENCIÓN COGNITIVA, AFECTIVAY SOCIAL, PARA LA MEJORA DE LOS PROCESOS FORMATIVOS DEL LICENCIADO EN EDUCACIÓN
}

\section{PROJECT OF COGNITIVE, AFFECTIVE AND SOCIAL INTERVENTION, FOR THE IMPROVEMENT OF THE EDUCATION DEGREE FOR ECUCATIONAL TRAINING PROCESSES}

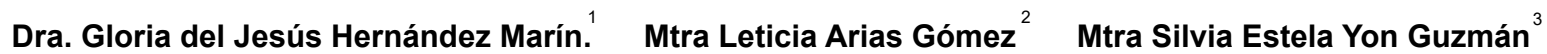
Facultad de Ciencias Educativas Universidad Autónoma del Carmen (UNACAR), México

$1 \quad$ Profesora investigadora de Tiempo Completo de la Facultad de Ciencias Educativas de la Universidad Autónoma del Carmen, entre sus últimas publicaciones de artículos científicos se encuentra: Autoevaluación del Liderazgo Pedagógico de los directores de secundarias, como apoyo a la mejora educativa, así como El currículo universitario por el enfoque de competencia, su pertinencia y nivel de exigencia en el contexto nacional y local en revista IJERI: International Journal of Educational Research and Innovation.

2 Leticia Arias Gómez, profesora investigadora de tiempo completo, miembro del Cuerpo Académico de Investigación en Humanidades de la Facultad de Ciencias Educativas en la Universidad Autónoma del Carmen (UNACAR), México. Es Licenciada en Pedagogía, hizo Especialidad en Docencia y Asesoría, realizó estudios de Maestría en Planeación de la Educación Superior. Entre sus publicaciones recientes se encuentran: Libro (2017), México en el Mundo. Competencia y ciudadanía en un entorno global pluricultural, Articulo Varela (2017): Estrategias de Aprendizaje para atender la diversidad en los estudiantes universitarios, Artículo REDIPE (2018): Habilidades digitales en la educación secundaria y su capacidad tecnológica instalada.

3 Profesora investigadora de Tiempo Completo Asociado C, desde 2011 es Gestora del Programa Educativo de la Licenciatura en Educación, de la Facultad de Ciencias Educativas de la Universidad Autónoma del Carmen. Estudió Master en Educación Especial e Integración escolar y social por la Universidad de Girona, España, Licenciada en Educación con especialidad en Defectología y especialización en Oligofrenopedagogía, por la Universidad Pedagógica Félix Varela, en Cuba. Es integrante del Cuerpo Académico en Consolidación Investigación en Humanidades, cuya línea de Investigación es: El currículo y los procesos formativos para el desarrollo del pensamiento complejo en educación y psicología. Es perfil PRODEP, autora de diversos capítulos de libros y artículos arbitrados e indizados, entre ellos, La inclusión de un estudiante con Trastorno del Espectro Autista en Educación Superior, (2018)., Los aspectos psicosociales en la construcción de un aprendizaje significativo en la educación básica: estudio de caso. (2018), Alcances y limitaciones del Programa de Tutoría en el nivel Superior. (2017), Hacia una experiencia significativa de aprendizaje a través de las TIC. (2016). La discapacidad es otro modo de capacidad. La interiorización del conocimiento. (2015). 


\section{RESUMEN}

El análisis de trayectoria de los alumnos del programa educativo de la Licenciatura en Educación, permitió generar un diagnóstico que sustenta la elaboración de la propuesta de intervención, que procura con sus estrategias, hacer uso de los principios y recursos para fortalecer el acompañamiento de los estudiantes, propuesto en el Programa Institucional de Tutoría (PIT). Su diseño atiende las dimensiones: afectiva, cognitiva y social por medio de talleres dirigidos a estudiantes, en tutoría grupal multigrado, durante el Horario Institucional de Tutoría (HIT), que desarrollan en colaborativo los profesores - tutores.

\section{PALABRAS CLAVE}

Tutoría, intervención, procesos formativos.

\section{ABSTRAC}

The analysis of trajectory of the students of the educational program of the Licenciatura en Educación, allowed to generate a diagnosis that supports the elaboration of the proposal of intervention, which seeks with its strategies, make use of the principles and resources to strengthen the accompaniment of the students, Proposed in the Programa Institucional de Tutoría (PIT). Its design takes care of the dimensions: affective, cognitive and social through workshops directed to students, in multi - group tutoring, during the Institutional Horario Institucional de Tutoria (HIT), developed by teachers - tutors.

\section{KEYWORTHS}

Tutoring, intervention, formative processes

\section{INTRODUCCIÓN}

El proyecto surge de la necesidad de dar respuestas a una problemática del Programa Educativo de la Licenciatura en Educación, respecto a la reprobación escolar de un $91 \%$ y de deserción de un $38 \%$ en las generaciones del plan de estudio 2010. Como parte de la función docente se encuentra la tutoría, que está establecida en el reglamento académico del profesorado en la Universidad Autónoma del Carmen, es por lo que el Grupo Disciplinar Investigación en Humanidades (GRUDIH), hizo suya esta propuesta a partir del análisis de los tutorados de los docentes del grupo. Este documento consta en su primera parte de un diagnóstico, que comprende la caracterización de tutorados y la evaluación de las necesidades vistas en el análisis de su trayectoria escolar, tomando como referencia los sistemas institucionales de datos al que acezan los docentes tutores y el gestor del programa educativo. El planteamiento del problema integra las siguientes interrogantes: ¿Qué estrategias coadyuvarían a la reducción de la reprobación y una mejor trayectoria escolar en los tutorados?; ¿Cómo puede influir el profesor tutor en los tutorados, para prevenir los problemas de reprobación escolar?

En su segunda parte el Plan de Intervención Tutorial, consta de dimensiones, objetivos, estrategias, actividades, acciones, metas, tiempos, recursos, cronograma para la implementación y acciones de evaluación, con el propósito de fortalecer el proceso formativo de los tutorados, con estrategias que atiendan las dimensiones: social, afectiva y cognitiva para mejorar la trayectoria escolar exitosa de los Licenciados en Educación.

\section{CARACTERIZACIÓN DE LOS TUTORADOS DE LA LICENCIATURA EN EDUCACIÓN}

Tomando como referencia la base de datos del Sistema Institucional de Tutoría (SIT-web, 2017) se realizó la caracterización de los tutorados, afín de generar un diagnóstico sociodemográfico, con las dimensiones sociales: edad, género, estado civil, dependencia económica, estatus económico (ingresos), lugar de procedencia, tipo de casa (rentada o propia), estado de salud y escolaridad de los padres. 
De los 42 tutorados, 12 (28\%) están casados o viven en pareja, 30 solteros que representa el $71 \%$. De ellos, $9(21 \%)$ son padres de familia, solo el $9 \%$ pertenecen al género masculino, el promedio de edad oscila de 20 a 25 años. El $13 \%$ de los estudiantes son foráneos en su mayoría de la región sur- sureste de México.

Se observa que 32 de los estudiantes, habitan en casa propia mientras que 10 de ellos rentan vivienda, respecto a su estatus económico 33 de los estudiantes respondieron que tienen un ingreso familiar mensual, en un rango de 1- 3 salarios $(\$ 1,517.00$ a $\$ 4,551.00), 7$ casos están en un rango de 4 - 8 salarios equivalente a $\$ 6,068.00$ a $\$ 12,136.00$, un caso tiene un rango 9 -12 salarios que equivalen a $\$ 13,653$ a $\$ 18,204$ y hay otro con un ingreso familiar de 16 salarios en adelante. Respecto al cuestionamiento sobre dependencia económica, 33 de los alumnos comentaron que lo hacen de sus padres, 4 son apoyadas por sus cónyuges y 4 trabajan para mantenerse en sus estudios, solo un caso no manifestó su dependencia.

En lo relacionado al estado de salud, 39 de los alumnos manifiestan encontrarse sanos y tres casos presentan complicaciones de salud entre las que se encuentran: epilepsia, migraña e hipotensión.

En la categoría de escolaridad de padres de toma en cuenta tanto los efectuados por la madre como por el padre, en el caso de la madre se identificó a $21(50 \%)$ con nivel básico de estudios, con nivel medio superior 14 (33\%), con nivel Licenciatura 3 casos $(7 \%$ ) y sin escolaridad $4(9 \%)$. En lo que refiere al padre $26(61 \%)$ de ellos cuenta con estudios de nivel básico, 8 $(19 \%)$ con nivel medio superior, con licenciatura $7(16 \%)$ y sin estudios, un caso $(2 \%)$.

Con los datos obtenidos podemos resumir, que de los 42 tutorados, la mayoría son solteros y habitan en casa propia, con estatus económico bajo en 33 de los estudiantes, en un rango de
1-3 salarios, son en su mayoría dependientes económicos de sus padres y solo 4 trabajan para mantenerse en sus estudios. Respecto al estado de salud, 39 de los estudiantes manifiestan encontrarse sanos y tres casos presentan complicaciones de salud que repercuten en el rendimiento académico: epilepsia, migraña e hipotensión.

\section{EVALUACIÓN DE NECESIDADES DE TRAYECTORIA ESCOLAR}

De acuerdo con los datos obtenidos se procede al análisis de las estrategias a desarrollar, para tal fin se concentra en la Tabla 1 los puntos de coincidencia por generación con los que se sustenta el diseño del plan. 
Tabla 1

Cursos con mayor incidencia de rezago por generación

\begin{tabular}{|c|c|c|c|c|}
\hline & Tutorados & $\begin{array}{l}\text { No. } \\
\text { alumnos } \\
\text { con rezago }\end{array}$ & \begin{tabular}{|l} 
No. de Cursos \\
rezagados
\end{tabular} & Cursos de rezago de mayor incidencia \\
\hline $2008-2012$ & 1 & 1 & 5 & 0 \\
\hline 2012 - 2016 & 11 & 11 & 17 & $\begin{array}{l}\text { Inglés } \\
\text { Seminario de investigación cuantitativa } \\
\text { Asesoría e intervención educativa } \\
\text { Prácticas Profesionales } \\
\text { Práctica y Análisis del trabajo docente }\end{array}$ \\
\hline $2013-2017$ & 1 & 1 & 1 & 0 \\
\hline $2014-2018$ & 10 & 7 & 41 & $\begin{array}{l}\text { Ingles } \\
\text { Estadística descriptiva }\end{array}$ \\
\hline $2015-2019$ & 13 & 7 & 17 & $\begin{array}{l}\text { Ingles } \\
\text { Estadística descriptiva } \\
\text { Razonamiento lógico } \\
\text { Bases epistemologías } \\
\text { Modelos curriculares } \\
\end{array}$ \\
\hline $2016-2020$ & 6 & 0 & 0 & \\
\hline Totales & 42 & 27 & * & 10 \\
\hline
\end{tabular}

*En este indicador existen coincidencias con respecto a los cursos que se reprueban por las diferentes generaciones, por lo que no se realiza sumatoria, tal es el caso del curso de inglés que se repite en diferentes niveles y generaciones.

El plan de estudio del Licenciado en Educación se conforma de 43 cursos que condicionan la trayectoria escolar adicionalmente el Servicio Social y Actividades de Formación Integral. De los 42 tutorados el $64 \%$ se encuentra en situación de rezago escolar, la generación que presenta mayor incidencia en el número de cursos con rezago es la 2014 - 2018, los cursos en los que se incide son: inglés y Estadística Descriptiva. Entre las causas por las cuales se da el rezago en los cursos mencionados esta la deserción, en muchos de los casos por abandono de los cursos desde la primera secuencia de aprendizaje, alumno se inscribe llega los primeros días de clase y posteriormente se ausenta y reprueba con un mínimo de calificación.

En la tabla anterior se puede apreciar que el $100 \%$ de los tutorados de las generaciones 2008, 2012, 2013 se encuentran rezagados, en la generación 2014 corresponde al 70\%, disminuyendo en la generación 2015 con el $53 \%$, pero aún es alto este índice de rezago, por lo que la muestra de estudiantes tiene un estimado entre cinco y seis años y medio para concluir sus estudios. 


\section{PLANTEAMIENTO DEL PROBLEMA}

Según datos tomados del Sistema Universitario de Control Escolar, (SUCE+), proporcionados por el gestor del programa educativo, el índice de retención (IR) del programa educativo de la Licenciatura en Educación en el plan de estudio 2010 es alto, el comportamiento que se presenta es como sigue: en las generaciones 2010 - 2014 es de un IR de $57 \%$, con un índice de deserción de un $43 \%$, cerca de la mitad de los que ingresaron, invirtiéndose los términos, en la generación 2011-2015, con el IR del $43 \%$, y una deserción de un $57 \%$, es decir mayor de la mitad, ambas generaciones con índices altos de deserción, las generaciones que están en activo son 20122016, lleva un índice de $72 \%$ de retención, con un índice de deserción de $28 \%$, disminuyendo significativamente; para 2013-2017 el IR es de $48 \%$, con una deserción de un $52 \%$, volviendo a incrementarse, en la generación 2014-2018, su índice de retención es de $81 \%$, disminuyó la deserción y se encuentra en un $19 \%$, la más baja de todas las generaciones, y la 2015-2019, es de $76 \%$, ligeramente con un incremento de $5 \%$ que la generación anterior. En promedio general se tiene un $61 \%$ de índice de retención en las 6 generaciones que se presentan y de deserción un $38 \%$, se manifiesta mayormente entre segundo y quinto semestre, lo mismo que el rezago y reprobación.

Las cifras anteriores nos hacen retomar a Delors (1996), quien enfatiza en la importancia $y$ transcendencia que tiene el ingreso y permanecía de los jóvenes a la universidad y es por lo que expone lo siguiente, entre el egreso del nivel básico, medio superior y la incorporación a la vida activa o el ingreso en la enseñanza superior, se decide el destino de millones de jóvenes. En este sentido exterioriza que el punto débil de los sistemas educativos, por una parte, es un exceso de elitismo, por otra parte, porque no se logra canalizar fenómenos de masificación o porque pecan de inercia y son refractarios a cualquier adaptación. Enfatiza que es la etapa donde los jóvenes se enfrentan con los problemas de la adolescencia, y consideran que están maduros pero en realidad sufren de una falta de madurez y el futuro suscita en ellos más ansiedad que despreocupación, por lo que es importante ofrecerles lugares de aprendizaje y de descubrimiento, proporcionarles los instrumentos necesarios para pensar y preparar su porvenir, así como diversificar las trayectorias en función de sus capacidades, y asegurar que existan perspectivas de futuro, donde puedan contar con la posibilidad de reparar los errores o corregir el rumbo.

Retomando el análisis anterior, se puede concluir que la matrícula desde 2010 hasta 2015 presenta un $38 \%$ de abandono, manifestado mayormente en 1 ro y 2 do año, un indicador elevado con respecto al ingreso; es de destacar que en la generación 2012 se incrementó la deserción en quinto semestre, correspondiente al $6.6 \%$ del valor promedio de todas las generaciones, revisando el indicador de reprobación escolar se aprecia que es alta en estas generaciones, con un $91 \%$. En la generación 2011 , hubo un incremento de la reprobación en un $41 \%$, luego descendió a $15 \%$ en el 2012, incrementándose en un $14 \%$ en 2013 , así como la deserción; entre los cursos de mayor reprobación se encuentran inglés, razonamiento lógico, política educativa y principios de administración.

El alto índice de reprobación escolar, rezago en las generaciones 2011, 2012 y 2013 es coincidente con un alto índice de deserción, ante esta situación que se presenta en el programa educativo, es que surge el interés establecer programas o estrategias que puedan ser atendidas estas necesidades. Es por lo que se consideró por el Grupo disciplinar Investigación en humanidades tomar una muestra de estudiantes, a partir de los tutorados que son atendidos por los profesores que conforman el grupo para implementar un programa de apoyo 
a los estudiantes objeto de análisis.

En el grupo seleccionado de 42 tutorados se evidencia que sus tienen un bajo nivel de formación profesional, la mayoría de los tutorados tienen dependencia económica de su padre, aun cuando tiene pareja o son madres solteras. El nivel de ingreso familiar mensual de más del $70 \%$ de los alumnos, oscila entre uno y tres salarios mínimos equivalentes a un máximo de $\$ 4,551.00$, evidenciando que tienen bajos ingresos, y en consecuencia disponen de menos recursos económicos para satisfacer sus necesidades básicas y por ende afrontar sus estudios, es de destacar que según Maslow, citado por Elizalde; Martí; Martínez (2006) la necesidad es la falta de algo, estas pueden ser: Necesidades deficitarias o inferiores, entre las que se encuentran: necesidades fisiológicas; necesidad de seguridad; de amor y de pertenencia; y de estima; si se presenta una variabilidad o carencias en ellas se pueden generar problemas psicológicos y/o fisiológicos. También están las Necesidades de desarrollo o superiores, orientadas hacia el logro de la autorrealización, este autor planta que ellas no son tan poderosas como las necesidades fisiológicas; por lo que pueden dañarse o perder su orientación más fácilmente que las necesidades primarias y demandan de elevadas influencias exteriores.

Entendiendo el planteamiento de este autor y su teoría el individuo tiene que satisfacer las necesidades básicas para sentirse motivado o impulsado a satisfacer necesidades superiores. Según Maslow, las necesidades básicas son más potentes y tienen prevalencia sobre las otras. Una vez satisfechas, se manifiestan las necesidades superiores y la persona se motiva para satisfacerlas, además estas necesidades cambian de persona a persona, porque cada individuo tiene sus propias características biológicas y mentales propias, además de códigos cultuales, familiares, regionales $\mathrm{o}$ locales entre otros.

En el caso de estos estudiantes apreciamos que el poder adquisitivo es limitado, pudiendo modificarse o ser variables la satisfacción de las necesidades fisiológicas; necesidad de seguridad; y de estima, ya que la universidad exige de gastos adicionales a los que comunes y estos pudieran estar en algún momento en peligro, por lo que constituyen un factor de riesgo para la continuidad de su formación profesional, afectando más, a un grupo que a otros, independientemente de tener similitudes de condiciones, ya que en los tutorados objetos de intervención, existen estudiantes con trayectoria regular, como con trayectoria irregular y reprobación.

Retomando a Maslow, citado por Elizalde; Martí; Martínez (2006), este asigna un orden jerárquico a las necesidades, ocupando el primer lugar las necesidades fisiológicas (básicas) y más potentes de todas, pero son las que tienen menor significado para la persona en busca de la autorrealización. Entre se ellas se encuentran la necesidad de liberarse de la sed y del hambre; de aliviar el dolor, el cansancio y el desequilibrio fisiológico; la necesidad de dormir, de sexo. Si las necesidades fisiológicas son satisfechas, o no constituyen un problema serio para la persona.

Segundo lugar, las necesidades de seguridad, que son la fuerza que domina la personalidad, entre las que se encuentran la preocupación por ahorrar, por comprar bienes y seguros, para obtener una vida ordenada, cierta, y un futuro predecible, en el que no existan amenazas para la integridad personal o familiar, pudiendo manifestarse temor y miedo, cuando está en riesgo.

Como terceras necesidades, se presentan el amor y pertenencia que representan la voluntad de reconocer y ser reconocido por los semejantes, de sentirse arraigados en lugares e 
integrados en redes y grupos sociales. en ellas están la necesidad de amigos, de compañeros, de una familia, de identificación con un grupo y de intimidad con una pareja. El éxito de lograr estas necesidades está en que las necesidades fisiológicas y de seguridad, estén satisfechas en cierto modo.

La cuarta necesidad la ocupa la estima, como necesidad psicológica y es importante satisfacerla para la evaluación personal y el reconocimiento de uno mismo, con respecto a los demás, se describen necesidad de respeto, de confianza basada en la opinión de otros, de admiración, de confianza en sí mismo, de auto valía y de auto aceptación, las carencias en ellas generan sentimientos de inferioridad que se manifiesta como vivencias de vergüenza o de culpa.

Como quinto, la ocupan las necesidades de autorrealización o metanecesidades, ubicadas en el segundo bloque de necesidades superiores o más subjetivas. Ellas varían de un individuo a otro, e incluye la autorrealización en todos los aspectos. Estando las necesidades de satisfacer nuestras propias capacidades personales, de desarrollar nuestro potencial, de hacer aquello para lo cual tenemos mejores aptitudes y la necesidad de desarrollar y ampliar los metamotivos (descubrir la verdad, crear belleza, producir orden y fomentar la justicia)

Además, están las necesidades cognitivas y las estéticas, según Maslow, citado por Elizalde; Martí; Martínez (2006), provienen de las necesidades básicas, necesidad cognitiva de todo ser humano normal de saber y comprender. La insatisfacción de ellas conduce a la frustración y al egoísmo. Las necesidades estéticas están relacionadas con el orden, la simetría y el cierre, la necesidad de aliviar la tensión producida por una labor no terminada y la necesidad de estructurar hechos, por lo que las circunstancias, ambientes agradables y hermosos favorecen el desarrollo de las personas.
Según Maslow, la insatisfacción de las necesidades trae consecuencias negativas para la persona, pues genera estados de frustración, egoísmo y su desarrollo se estanca. Para resolver la situación problema a que nos enfrentamos, nos hacemos las siguientes interrogantes:

¿Qué estrategias coadyuvarían a la reducción de la reprobación y una mejor trayectoria escolar en los tutorados?

¿Cómo puede influir el profesor tutor en los tutorados, para prevenir los problemas de reprobación escolar?

Para ello, se propone un plan de intervención que contemple estrategias que atiendan las dimensiones: social, afectiva y cognitiva, tomando en consideración los planteamientos realizados por Maslow.

\section{PLAN DE INTERVENCIÓN TUTORIAL}

En este plan de intervención participan cuatro profesores tutores e investigadores del Grupo Disciplinar Investigación en Humanidades de la Facultad de Ciencias Educativas, y que atienden directamente el programa educativo, participan además 42 estudiantes tutorados de estos profesores, de las generaciones 2008, 2012, 2013, 2014, 2015 y 2016, consta de un objetivo general, un programa de intervención que contiene dimensiones, objetivos, estrategias, actividades, acciones, metas, tiempos, recursos, cronograma para la implementación y acciones de evaluación. Se inicia en febrero y concluye en el mes de junio, de acuerdo al ciclo escolar, lo conforman 12 talleres que actuarán como tutoría correctiva en las generaciones 2008 a 2014 y predictiva en las recientes, 2015 y 2016 para la atención de las dimensiones, que aparecen a continuación. 
Tabla 2

\section{Programa de intervención}

\begin{tabular}{|l|l|}
\hline Dimensiones & $\begin{array}{l}\text { Áreas de la orientación. } \\
\text { (Bisquerra 2012) }\end{array}$ \\
\hline Social & Orientación profesional \\
\hline Afectiva & $\begin{array}{l}\text { Prevención y desarrollo } \\
\text { humano }\end{array}$ \\
\hline Cognitiva & $\begin{array}{l}\text { Orientación en los procesos } \\
\text { de aprendizaje }\end{array}$ \\
\hline
\end{tabular}

Según Bisquerra (2012) entre las áreas de la orientación se encuentran: la orientación profesional que implica adquirir autonomía de información, toma de decisiones, competencias socio-emocionales, para la búsqueda del empleo, estrategias de afrontamiento de situaciones críticas (conflictos, desempleo, etc), aprender a aprender para progresar profesionalmente a lo largo de toda la vida, aprender a emprender, es preparar para la vida.
La orientación en los procesos de aprendizaje, contempla estrategias de aprendizaje y temas afines tales como métodos y técnicas de estudio, habilidades de aprendizaje, aprender a aprender y está muy vinculada con las necesidades específicas de apoyo educativo, en las que se encuentran los grupos en riesgo y desfavorecidos.

La tercera área de la orientación, que será atendida es la prevención y desarrollo humano, se encarga fundamentalmente del aprender a ser.

\section{OBJETIVO GENERAL:}

Fortalecer el proceso formativo de los tutorados, con estrategias que atiendan las dimensiones: social, afectiva y cognitiva para la mejora de la trayectoria escolar de los Licenciados en Educación

Tabla 3

Programa de intervención

\begin{tabular}{|c|c|c|c|c|c|c|}
\hline Dimensión & Estrategia & Acción & Meta & Tiempo & Responsables & Recursos \\
\hline Afectiva & $\begin{array}{l}\text { Favorecer la } \\
\text { educación } \\
\text { emocional para } \\
\text { aprender a ser. }\end{array}$ & $\begin{array}{l}\text { Aplicar } \\
\text { diagnóstico del } \\
\text { auto concepto y } \\
\text { la autoestima. } \\
\text { Desarrollo } 4 \\
\text { talleres con } \\
\text { actividades para } \\
\text { la educación } \\
\text { emocional. }\end{array}$ & $\begin{array}{l}\text { Que los } \\
\text { tutorados } \\
\text { aprendan a } \\
\text { educar su } \\
\text { autoestima } \\
\text { Desarrollar la } \\
\text { habilidad de } \\
\text { auto motivarse } \\
\text { Adoptar una } \\
\text { actitud positiva } \\
\text { ante la vida. }\end{array}$ & 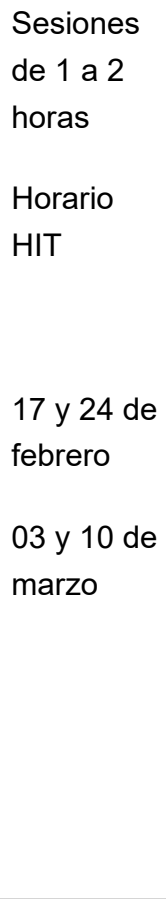 & $\begin{array}{l}\text { Mtra. Silvia } \\
\text { Estela Yon } \\
\text { Guzmán y Dra. } \\
\text { Sara E. Castillo } \\
\text { Ortega }\end{array}$ & $\begin{array}{l}\text { Listado de } \\
\text { adjetivos de } \\
\text { significado } \\
\text { opuesto. } \\
\text { Hoja de trabajo; } \\
\text { siento y pienso. } \\
\text { Recortes de } \\
\text { imágenes de } \\
\text { prensa donde } \\
\text { aparezcan } \\
\text { personas con } \\
\text { expresiones } \\
\text { emocionales. } \\
\text { Hoja de trabajo. } \\
\text { Emociones; } \\
\text { causas y } \\
\text { consecuencias. }\end{array}$ \\
\hline
\end{tabular}




\begin{tabular}{|c|c|c|c|c|c|c|}
\hline Cognitiva & $\begin{array}{l}\text { Impulsar el uso } \\
\text { de estrategias } \\
\text { de retención, } \\
\text { comprensión y } \\
\text { uso activo del } \\
\text { conocimiento. }\end{array}$ & $\begin{array}{l}\text { Generar } \\
\text { diagnóstico } \\
\text { de dominio de } \\
\text { estrategias. } \\
\text { Desarrollo } \\
\text { de atención } \\
\text { individualizada } \\
\text { y grupal con los } \\
\text { tutorados. }\end{array}$ & $\begin{array}{l}\text { Aplicación de } 4 \\
\text { talleres } \\
\text { Validación } \\
\text { de cuatro } \\
\text { estrategias } \\
\text { cognitivas } \\
\\
\text { Reporte de } \\
\text { evaluación de } \\
\text { impactos en } \\
\text { la aplicación } \\
\text { de estrategias } \\
\text { cognitivas. }\end{array}$ & 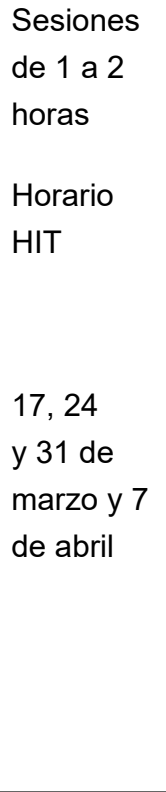 & $\begin{array}{l}\text { Dra. Gloria del } \\
\text { J. Hernández } \\
\text { Marín }\end{array}$ & $\begin{array}{l}\text { Manuales } \\
\text { laboratorio de } \\
\text { aprendizaje, } \\
\text { cañón, } \\
\text { computadora } \\
\text { fotocopiado de } \\
\text { materiales } \\
\text { plumones de } \\
\text { color } \\
\text { materiales } \\
\text { didácticos }\end{array}$ \\
\hline Social & $\begin{array}{l}\text { Promover la } \\
\text { autovaloración } \\
\text { de las } \\
\text { habilidades } \\
\text { interpersonales }\end{array}$ & $\begin{array}{l}\text { Aplicación de } \\
\text { instrumentos } \\
\text { de auto } \\
\text { diagnóstico. } \\
\\
\text { Desarrollo de } \\
\text { actividades con } \\
\text { los participantes } \\
\text { e invitados que } \\
\text { fortalezcan } \\
\text { su conducta } \\
\text { y relaciones } \\
\text { interpersonales. }\end{array}$ & $\begin{array}{l}\text { Diagnóstico } \\
\text { de liderazgo al } \\
100 \% \text { de los } \\
\text { tutorados. } \\
\text { Desarrollo de } \\
\text { cuatro talleres } \\
\text { que fortalezcan } \\
\text { la convivencia } \\
\text { social entre } \\
\text { participantes y } \\
\text { con su contexto } \\
\text { Reporte de } \\
\text { evaluación de } \\
\text { impactos en } \\
\text { la aplicación } \\
\text { de estrategias } \\
\text { interpersonales. }\end{array}$ & $\begin{array}{l}\begin{array}{l}\text { Sesiones } \\
\text { de } 1 \text { a } 2 \\
\text { horas }\end{array} \\
\text { Horario } \\
\text { HIT }\end{array}$ & $\begin{array}{l}\text { Mtra. Leticia } \\
\text { Arias Gómez }\end{array}$ & $\begin{array}{l}\text { Cañón / } \\
\text { computadora } \\
\text { Papelería } \\
\text { Copias de } \\
\text { cuestionarios y } \\
\text { programa }\end{array}$ \\
\hline
\end{tabular}

El día 10 de febrero se efectúa la sesión de presentación y de bienvenida al programa de intervención tutorial, el 02 de junio corresponde al cierre, presentación de portafolios y evaluación de impacto del proyecto.

\section{METODOLOGÍA DE EVALUACIÓN DE IMPACTOS}

A fin de hacer seguimiento a los resultados de cada sesión, dimensión y de la estrategia completa se estructuran los siguientes eventos de evaluación: 


\begin{tabular}{|l|}
\hline Actividades de evaluación \\
\hline Portafolio de los tutorados con las actividades realizadas en las sesiones de tutoría. \\
\hline Valoración de las actividades realizadas por cada tutor (durante y al cierre de la dimensión. \\
\hline Evaluación por los alumnos, individual y colectiva, de las diferentes actividades. \\
\hline Evaluación y redacción del reporte final \\
\hline
\end{tabular}

La estrategia está en proceso de acuerdo con el cronograma programado contando con la participación entusiasta de los tutores y tutorados, al cierre del ciclo se procesará a la integración y valoración de los resultados.

\section{REFERENCIAS BIBLIOGRÁFICAS}

Bisquerra (2012). Orientación, tutoría y educación emocional. Madrid. España: Síntesis.

Corral, V. y Díaz, X. (s.f.) Factores Asociados a la reprobación de los estudiantes de la Universidad de Sonora. Recuperado de http://www.comie. org. $\mathrm{mx} /$ congreso/memoriaelectronica/v10/pdf/ area_tematica_16/ponencias/0910-F.pdf

Delors, J. (1996). Informe a la UNESCO de la Comisión Internacional sobre la Educación para el siglo XXI. La educación encierra un tesoro. Recuperado de http://www.unesco.org/ education/pdf/DELORS_S.PDF

Elizalde, A., Martí, M. y Martínez, F. A. (2006). Una revisión crítica del debate sobre las necesidades humanas desde el Enfoque Centrado en la Persona. POLIS, Revista Latinoamericana: [Fecha de consulta: 19 de enero de 2017] Recuperado de http://www.redalyc.org/articulo. oa? $\mathrm{id}=30517306006>$ ISSN 0717-6554 